\title{
Coherent manipulation of three-qubit states in a molecular single-ion magnet
}

\author{
M. D. Jenkins, ${ }^{1,2}$ Y. Duan, ${ }^{3}$ B. Diosdado, ${ }^{4}$ J. J. García-Ripoll, ${ }^{5}$ A. Gaita-Ariño, ${ }^{3}$ C. Giménez-Saiz, ${ }^{3}$ P. J. Alonso,,${ }^{1,2 *}$ \\ E. Coronado, ${ }^{3, \dagger}$ and F. Luis ${ }^{1,2, \ddagger}$ \\ ${ }^{1}$ Instituto de Ciencia de Materiales de Aragón (ICMA), CSIC-Universidad de Zaragoza, Pedro Cerbuna 12, 50009 Zaragoza, Spain \\ ${ }^{2}$ Dpto. de Física de la Materia Condensada, Universidad de Zaragoza, Pedro Cerbuna 12, 50009 Zaragoza, Spain \\ ${ }^{3}$ Instituto de Ciencia Molecular (ICMol), Universidad de Valencia, Catedrático José Beltrán 2, 46980 Paterna, Spain \\ ${ }^{4}$ Servicio de Difracción de Rayos X y Análisis por Fluorescencia, Universidad de Zaragoza, 50009 Zaragoza, Spain \\ ${ }^{5}$ Instituto de Física Fundamental, IFF-CSIC, Serrano 113-bis, 28006 Madrid, Spain
}

(Received 13 October 2016; revised manuscript received 18 January 2017; published 22 February 2017)

\begin{abstract}
We study the quantum spin dynamics of nearly isotropic $\mathrm{Gd}^{3+}$ ions entrapped in polyoxometalate molecules and diluted in crystals of a diamagnetic $\mathrm{Y}^{3+}$ derivative. The full energy-level spectrum and the orientations of the magnetic anisotropy axes have been determined by means of continuous-wave electron paramagnetic resonance experiments, using X-band $(9-10 \mathrm{GHz})$ cavities and on-chip superconducting waveguides and $1.5-\mathrm{GHz}$ resonators. The results show that seven allowed transitions between the $2 S+1$ spin states can be separately addressed. Spin coherence $T_{2}$ and spin-lattice relaxation $T_{1}$ rates have been measured for each of these transitions in properly oriented single crystals. The results suggest that quantum spin coherence is limited by residual dipolar interactions with neighbor electronic spins. Coherent Rabi oscillations have been observed for all transitions. The Rabi frequencies increase with microwave power and agree quantitatively with predictions based on the spin Hamiltonian of the molecular spin. We argue that the spin states of each $\mathrm{Gd}^{3+}$ ion can be mapped onto the states of three addressable qubits (or, alternatively, of a $d=8$-level "qudit"), for which the seven allowed transitions form a universal set of operations. Within this scheme, one of the coherent oscillations observed experimentally provides an implementation of a controlled-controlled-NOT (or Toffoli) three-qubit gate.
\end{abstract}

DOI: 10.1103/PhysRevB.95.064423

\section{INTRODUCTION}

Molecular nanomagnets [1,2] are artificial molecules, designed and synthesized by chemical methods. They consist of a magnetic core surrounded by nonmagnetic ligands, are perfectly monodisperse, and remain stable in different material forms, from crystals to solutions and, in some cases, also when they are deposited onto solid substrates [3]. In the past two decades, they have shown to be model materials to investigate a plethora of magnetic quantum phenomena, such as spin tunneling [4-7] and coherence [8,9], quantum phase interference [10,11], spin entanglement [12], and quantum phase transitions [13]. More recently, they have emerged as candidates to embody spin qubits, the building blocks of future quantum computers [14-22]. In these systems, the main sources of magnetic noise, which introduce decoherence, arise from hyperfine couplings to nuclear spins and from dipolar couplings to other electronic spins $[23,24]$. These effects can be minimized by isotopical purification and by extreme dilution in a diamagnetic matrix or in appropriate solvents. This strategy has recently led to spin coherence times [16,20,21] comparable to those reported for nitrogen-vacancy centers in diamond and $\mathrm{P}$ donors in silicon $[25,26]$. However, it also limits studying quantum dynamics in well-defined systems consisting of several qubits.

An appealing alternative, which we explore in this work, is to embody $N>1$ qubits using $d=2^{N}$ internal spin levels of a molecular nanomagnet. Its practical implementation is not straightforward, though. In the case of polynuclear clusters,

\footnotetext{
*alonso@unizar.es

†eugenio.coronado@uv.es

†fluis@unizar.es
}

such as the well-known $\mathrm{Mn}_{12}$ acetate with a spin $S=10$ [14], spin coherence times are very short in concentrated crystals, while experiments in frozen solutions do not enable us to address each transition separately from the others. A possibility is to use simple nanomagnets made of just one lanthanide ion (also referred to as "single-ion magnets") that show sufficiently long coherence times in crystalline hosts $[22,27,28]$. However, the zero-field splitting associated with the crystal field interaction is often so large that only the lowest-lying electronic doublet is experimentally accessible. These difficulties can be overcome by a judicious choice of the lanthanide ion and a proper design of its environment. We focus on gadolinium that, because of its $4 f^{7}$ electronic configuration, has no net orbital moment $L$ but instead possesses the largest spin $S=7 / 2$ attainable by any single atom. The eight spin states span the Hilbert space of three qubits. This intrinsic property of any free $\mathrm{Gd}^{3+}$ ion is, however, not yet sufficient. A second necessary ingredient is the existence of a set of coherent transitions able to connect any two arbitrary states. For this, some magnetic anisotropy is required, sufficiently large to enable addressing each transition independently of the others but also sufficiently weak to guarantee that all transition frequencies lie within the range accessible by microwave technologies. A further advantage of $\mathrm{Gd}^{3+}$ is that zero field splittings are one to two orders of magnitude smaller than those found for other lanthanide or transition-metal ions. Furthermore, these splittings strongly depend on the local coordination, which opens subtle possibilities for design [18].

In this work, the system of choice is a molecular nanomagnet which can be formulated as $\left[\mathrm{Gd}\left(\mathrm{H}_{2} \mathrm{O}\right) \mathrm{P}_{5} \mathrm{~W}_{30} \mathrm{O}_{110}\right]^{12-}$. It is shown in Fig. 1(a). Using potassium as counterion, it forms crystals formulated as $\mathrm{K}_{12} \mathrm{Gd}\left(\mathrm{H}_{2} \mathrm{O}\right) \mathrm{P}_{5} \mathrm{~W}_{30} \mathrm{O}_{110} \cdot 27.5 \mathrm{H}_{2} \mathrm{O}$, referred to hereafter as $\mathrm{GdW}_{30}$. In this molecule, $\mathrm{Gd}^{3+}$ is 
(a)
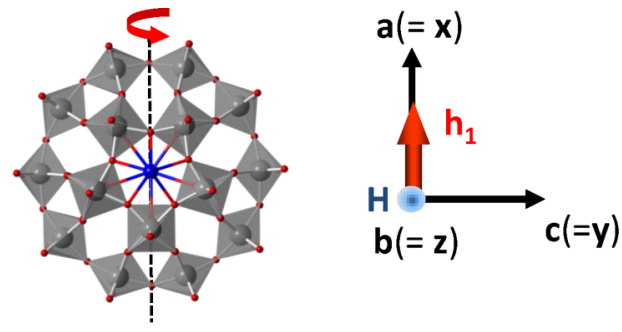

(b)

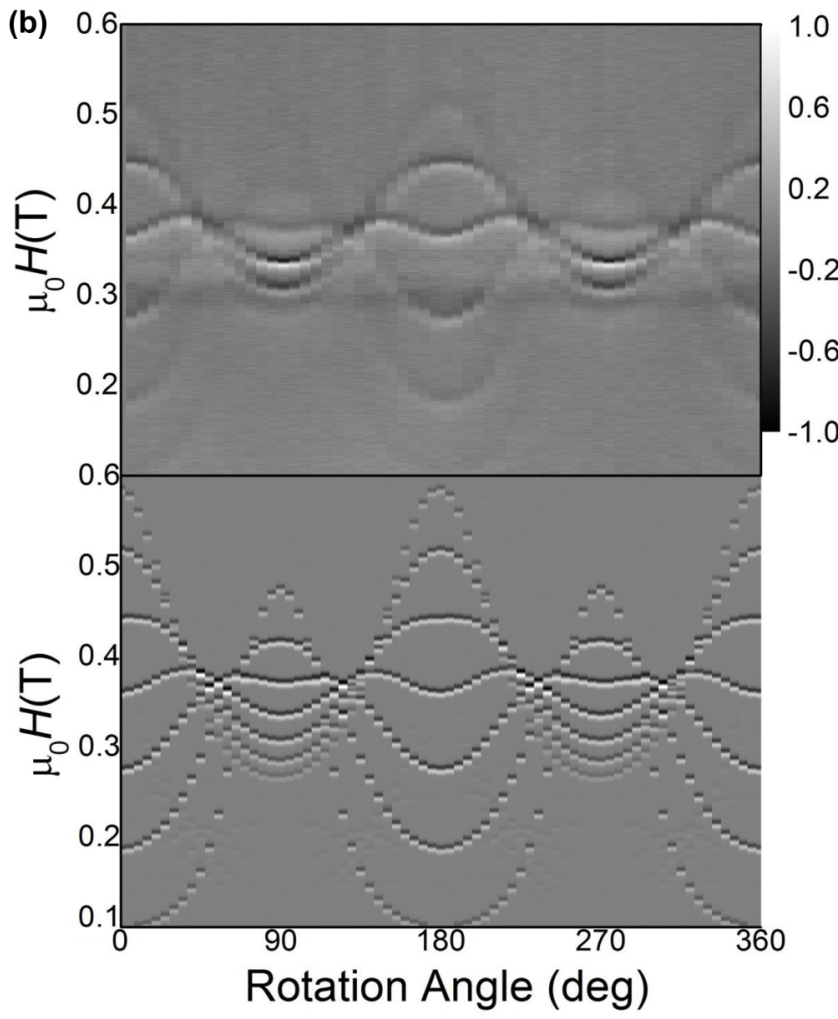

FIG. 1. (a) Molecular structure of a $\mathrm{GdW}_{30}$ POM cluster. The arrows show the approximate orientations of the hard $(z)$, medium $(x)$, and easy (y) magnetic axes and the experimental geometry used in the pulsed EPR experiments, where $\vec{H}$ denotes the static magnetic field and $\vec{h}_{1}(t)=2 h_{1} \cos (\omega t) \hat{x}$, with $\omega / 2 \pi=9.77 \mathrm{GHz}$ (X-band), is the microwave magnetic field. (b) Angle-dependent electron paramagnetic resonance spectra. Top panel: Rotational $\mathrm{X}$-band cw-EPR spectrum measured at room temperature on a single crystal of $\mathrm{Y}_{0.99} \mathrm{Gd}_{0.01} \mathrm{~W}_{30}$ that was rotated along its $a$ crystallographic axis. Bottom panel: Rotational spectrum calculated, for the same conditions, using the spin Hamiltonian (1) with the parameters given in the text.

encapsulated inside a "doughnut"-shaped polyoxometalate (POM) cluster and coordinated to 10 oxygen atoms and one apical water. The resulting coordination around $\mathrm{Gd}^{3+}$ has an unusual planar shape and symmetry close to $\mathrm{C}_{5 \mathrm{v}}$. This leads to an easy-plane spin anisotropy, with a hard magnetic axis along the main molecular axis $z$, and to an overall energy splitting of the $S=7 / 2$ multiplet smaller than $1 \mathrm{~K}(20.8 \mathrm{GHz})$.

The manuscript is organized as follows. Section II gives details of the sample preparation and characterization methods as well as of electron paramagnetic resonance (EPR) experiments. The results of these experiments are then described in Sec. III. They provide information on the magnetic energy- level structure and the magnetic states (Sec. III A) and on spin coherence and relaxation times (Sec. III B) and show that all allowed transitions can be coherently controlled (Sec. III C) and that they form a complete set for a three-qubit system (Sec. IIID). Finally, Sec. IV is devoted to summarize the results and discuss some of the prospects they open to future research.

\section{EXPERIMENTAL DETAILS}

\section{A. Synthesis}

Samples of $\mathrm{GdW}_{30}$ and of $\mathrm{K}_{12}\left[\mathrm{Y}\left(\mathrm{H}_{2} \mathrm{O}\right) \mathrm{P}_{5} \mathrm{~W}_{30} \mathrm{O}_{110}\right]$. $29 \mathrm{H}_{2} \mathrm{O}\left(\mathrm{YW}_{30}\right.$ ) were prepared by adaptations of previously reported methods [29]. Samples of magnetically diluted systems (potassium salts of $\left[\mathrm{Gd}_{x} \mathrm{Y}_{1-x}\left(\mathrm{H}_{2} \mathrm{O}\right) \mathrm{P}_{5} \mathrm{~W}_{30} \mathrm{O}_{110}\right]^{12-}$, referred to as $\mathrm{Y}_{1-x} \mathrm{Gd}_{x} \mathrm{~W}_{30}$ with $x=0.01$ and 0.05 ), were prepared by dissolving pure crystal samples of $\mathrm{GdW}_{30}$ and $\mathrm{YW}_{30}$ in the proper molar ratio at $80^{\circ} \mathrm{C}$. Crystals of $\mathrm{Y}_{1-x} \mathrm{Gd}_{x} \mathrm{~W}_{30}$ were obtained from these solutions after several days standing in open beakers at room temperature. The chemical compositions were evaluated by inductively coupled plasma-atomic emission spectroscopy.

\section{B. $x$-Ray diffraction}

Suitable crystals of $\mathrm{GdW}_{30}, \mathrm{YW}_{30}$, and $\mathrm{Gd}_{x} \mathrm{Y}_{1-x} \mathrm{~W}_{30}$ $(x=0.01$ and 0.05$)$ were taken from solution, coated with Paratone $\mathrm{N}$ oil, suspended on small fiber loops, and placed in a stream of cooled nitrogen $(120 \mathrm{~K})$ on an Oxford Diffraction diffractometer equipped with a graphite-monochromated enhanced (Mo) x-ray source $(\lambda=0.71073 \AA)$. For $\operatorname{GdW}_{30}$ and $\mathrm{YW}_{30}$, data collection routines, unit cell refinements, and data processing were carried out using the CRYSALIS software package developed by Agilent Technologies. The structure solution and refinement were carried out using SHELXS-97 and SHELXL-2014 [30]. The crystal structure and crystallographic data are shown in Ref. [31]. For $\mathrm{Gd}_{x} \mathrm{Y}_{1-x} \mathrm{~W}_{30}$ $(x=0.01$ and 0.05$) \mathrm{x}$-ray powder diffractograms indicate that all compounds have the same cell parameters [31] and are therefore isostructural. $\mathrm{x}$-Ray diffraction experiments were also performed on single crystals of $\mathrm{Y}_{0.99} \mathrm{Gd}_{0.01} \mathrm{~W}_{30}$ [31]. The results are compatible with the orthorhombic crystal structure of the undiluted $\mathrm{GdW}_{30}$, with lattice parameters $a=28.814 \AA$, $b=21.411 \AA$, and $c=20.825 \AA$.

\section{Electron paramagnetic resonance experiments}

Continuous wave (cw) and pulsed time domain (TD) EPR measurements were performed with a Bruker Biospin ELEXSYS E-580 spectrometer operating in the X-band (9-10 $\mathrm{GHz}$ ). The temperature of the sample was varied between $6 \mathrm{~K}$ and $300 \mathrm{~K}$ using gas-flow helium cryostats. Polycrystalline samples were introduced in quartz tubes and sealed under an Ar atmosphere. Single crystals (typically $1 \times 0.3 \times 0.3 \mathrm{~mm}^{3}$ ) were oriented by $\mathrm{x}$-ray diffraction and glued on a $2 \times 2 \times 2 \mathrm{~mm}^{3}$ nylon cube with their $a$ crystallographic axis parallel to one of the cube's edges. The cube was mounted on a sample holder that can be rotated around the vertical laboratory axis in order to measure the angular dependence of the cw-EPR spectra and 
to get the desired orientation of the crystal with respect to the magnetic field for the TD experiments.

On-chip EPR experiments were performed by coupling single crystals of undiluted $\mathrm{GdW}_{30}$ to superconducting coplanar waveguides and $1.5-\mathrm{GHz}$ resonators. These devices are fabricated on 500- $\mu \mathrm{m}$-thick C-plane sapphire wafers and consist of a 150-nm-thick niobium layer deposited by radiofrequency sputtering. The $400-\mu \mathrm{m}$ central line and the $200-\mu \mathrm{m}$ gaps separating it from the ground planes were patterned by either photolithography and lift-off or reactive ion etching. The device and the sample were immersed in a liquid helium bath at $T=4.2 \mathrm{~K}$ inside the bore of a $9 \mathrm{~T} \times 1 \mathrm{~T} \times 1 \mathrm{~T}$ superconducting vector that enables controlling the magnitude and orientation of the external magnetic field.

\section{Spin nutation experiments}

The spin nutation signals of different $\mathrm{Y}_{0.99} \mathrm{Gd}_{0.01} \mathrm{~W}_{30}$ single crystals have been obtained by measuring the amplitude of the electron spin echo (ESE) generated by the application of a twopulse sequence: $p\left(t_{\mathrm{p}}\right)-\tau-p(\pi)-\tau-\mathrm{ESE}$ as a function of the first pulse length $t_{\mathrm{p}}$ [32]. In a particular experiment, the interval $\tau$ between the two pulses was kept fixed (typically 120 to $200 \mathrm{~ns}$ ) and the length of the $\pi$ pulse was adjusted to maximize the echo amplitude for each high-power attenuation (HPA). This pulse is also sufficiently short (less than $40 \mathrm{~ns}$ ) to ensure that the hard-pulse approximation describes well the spin dynamics taking place under its influence. The measured signal is then proportional to the $S_{y}\left(t_{\mathrm{p}}\right)$ spin component (in the rotating system) generated by the nutation pulse $p\left(t_{\mathrm{p}}\right)$. By contrast, the length $t_{\mathrm{p}}$ of the nutation pulse can significantly exceed $\mathrm{T}_{2}$. The ensuing spin dynamics and the actual pulse shapes must therefore be explicitly taken into account. The ideal description as a square-shaped pulse becomes a good approximation for sufficiently long pulses. However, for $t_{\mathrm{p}} \lesssim 100 \mathrm{~ns}$ the actual shape departs significantly from this description. For this reason, besides other considerations on the spectral excitation [31], the analysis of the nutation signal takes into account data measured for $t_{\mathrm{p}}$ larger than an onset $t_{m}$. Typically, $t_{m}=100 \mathrm{~ns}$. It has also been checked that the relative height of the "flat region" of the "in phase" excitation pulse follows the expected exponential dependence on HPA. Further details on the analysis of spin nutation signals are given as part of the supplementary material [31].

\section{RESULTS AND DISCUSSION}

\section{A. Single-crystal cw-EPR: Energy-level scheme and spin states}

The energy-level spectrum of $\mathrm{GdW}_{30}$ subject to a dc magnetic field $\vec{H}$ can be described by the spin Hamiltonian $[18,33]$,

$$
\mathcal{H}=D\left[S_{z}^{2}-\frac{1}{3} S(S+1)\right]+E\left(S_{x}^{2}-S_{y}^{2}\right)-g \mu_{B} \vec{S} \cdot \vec{H}
$$

where $D=1281 \mathrm{MHz}$ and $E=294 \mathrm{MHz}$ are second-order magnetic anisotropy constants, determined from the fit of cw-EPR spectra recorded on powder samples [18,31] and $g=2$ is the gyromagnetic ratio. The orientations of the magnetic axes $x, y$, and $z$ have been found by performing angle-dependent cw-EPR experiments on $\mathrm{Y}_{1-x} \mathrm{Gd}_{x} \mathrm{~W}_{30}$ single crystals. Representative results are shown in Fig. 1 and in Ref. [31]. It follows that $x, y$, and $z$ are close to the crystallographic axes $a, c$, and $b$, respectively. Additional spectroscopic information has been obtained by coupling $\mathrm{GdW}_{30}$ single crystals to superconducting coplanar wave guides and $1.5-\mathrm{GHz}$ resonators. The transmission through an open waveguide enables exploring, at each magnetic field, frequencies ranging from $0.01 \mathrm{GHz}$ to $14 \mathrm{GHz}$; thus it provides direct information on the full energy-level spectrum. Experiments of this kind performed at $T=4.2 \mathrm{~K}$ with $\vec{H}$ pointing close to $a$, thus to the medium magnetic anisotropy axis $x$, are shown in Fig. 2, while data obtained with a $1.5-\mathrm{GHz}$ superconducting resonator are shown in Ref. [31]. The results agree with simulations based on Eq. (1). Almost identical spectroscopic results are obtained for samples with different concentrations of $\mathrm{GdW}_{30}$, which shows that the anisotropy parameters and the ensuing energy-level scheme is an intrinsic property of each isolated molecule.

The magnetic anisotropy provides the anharmonicity that is required to address each transition individually but it is also sufficiently weak to make them accessible by conventional $\mathrm{X}$-band (9.48-GHz) electron paramagnetic resonance. In order to maximize the field splitting between these transitions and to make them easier to visualize, $\vec{H}$ was oriented close to the hard magnetic axis of the molecule $z$. This gives rise to the X-band cw-EPR spectrum shown in Fig. 3(a) and to the energy-level scheme shown in Fig. 3(b). Because of the weak magnetic anisotropy, the eigenstates $|n\rangle$ of the spin Hamiltonian (1) that are involved in the observed transitions are close to spin projections $|m\rangle$ along $\vec{H}$, with $m=-S+(n-1)$, and their energies $\epsilon_{n} \simeq D\left[3 m^{2}-S(S+1)\right]-g \mu_{\mathrm{B}} H_{z} m$.

The spread in the resonance frequencies $\omega_{n} \equiv\left(\epsilon_{n+1}-\epsilon_{n}\right) / h$ associated with transitions $n=1$ to 7 can be tuned by setting the magnetic field strength and its orientation (see Fig. 1). For instance, at $\mu_{0} H=0.325 \mathrm{~T}, \omega_{n}$ values range from $1.4 \mathrm{GHz}$ to $16.7 \mathrm{GHz}$ when $\vec{H} \| \vec{z}$, whereas they become much closer (between $7.1 \mathrm{GHz}$ and $10.2 \mathrm{GHz}$ ) if the field points instead along $x$, as can be seen in the results shown in Fig. 2 (see also Figs. S4- S6 in Ref. [31]).

\section{B. Spin coherence and spin relaxation}

The spin dynamics has been studied at low temperature $(T=6 \mathrm{~K})$ on magnetically diluted $\mathrm{Y}_{0.99} \mathrm{Gd}_{0.01} \mathrm{~W}_{30}$ single crystals, in which $99 \%$ of $\mathrm{GdW}_{30}$ molecules have been replaced with its diamagnetic $\mathrm{YW}_{30}$ equivalent to reduce dipolar interactions. Phase coherence times $\mathrm{T}_{2}$ have been determined by measuring the spin echo following a Hahn sequence of $\pi / 2$ and $\pi$ pulses resonant with a given transition and separated by a varying time interval $\tau$ [31]. The magnetic field dependence of the echo amplitude [Fig. 4(a)] shows resonances that closely match those found in the continuous EPR spectrum measured under similar conditions. These results show that all seven transitions can be coherently manipulated. Different transitions show very similar coherence times [Fig. 4(b)], ranging approximately between 470 and $600 \mathrm{~ns}$.

The nuclear spin bath of a $\mathrm{GdW}_{30}$ molecule is formed by the protons of water molecules $(100 \%, I=1 / 2)$, and the nuclear spins of the $\mathrm{P}(100 \%, I=1 / 2), \mathrm{K}(100 \%, I=3 / 2)$, and $\mathrm{W}(14 \%, I=1 / 2)$ isotopes. Recent calculations show, 


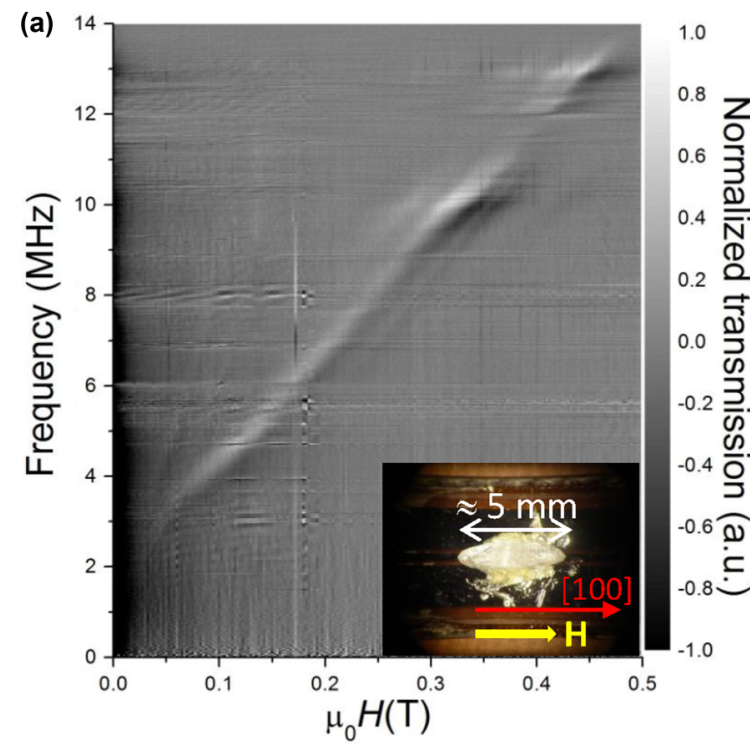

(c)

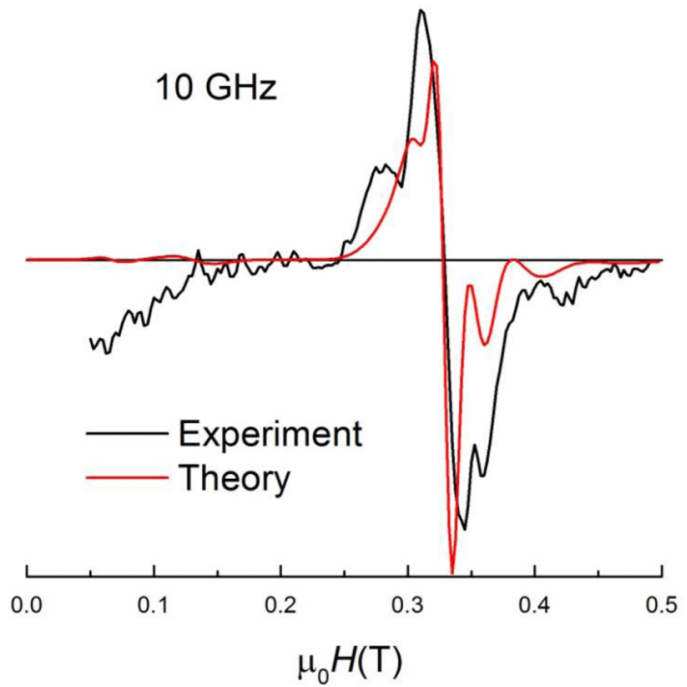

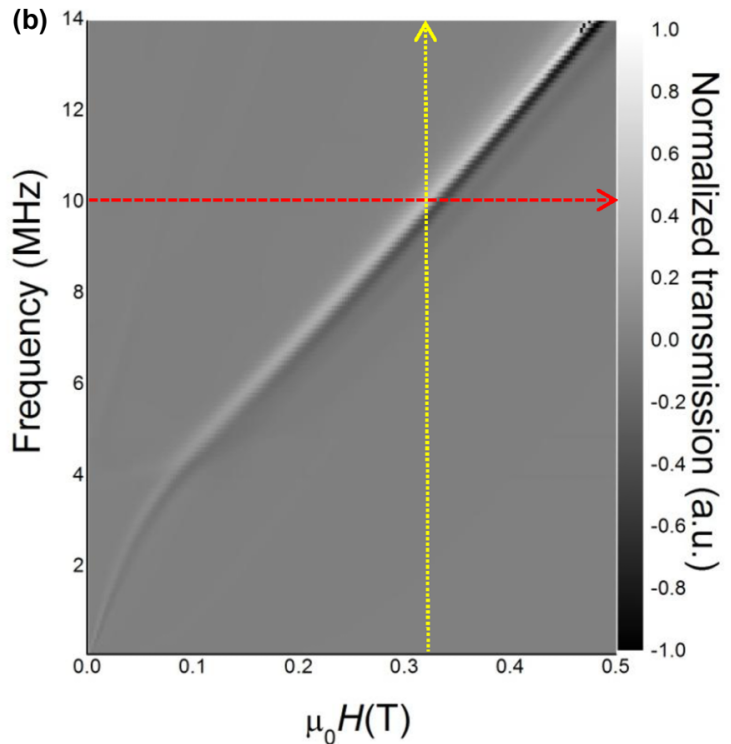

(d)

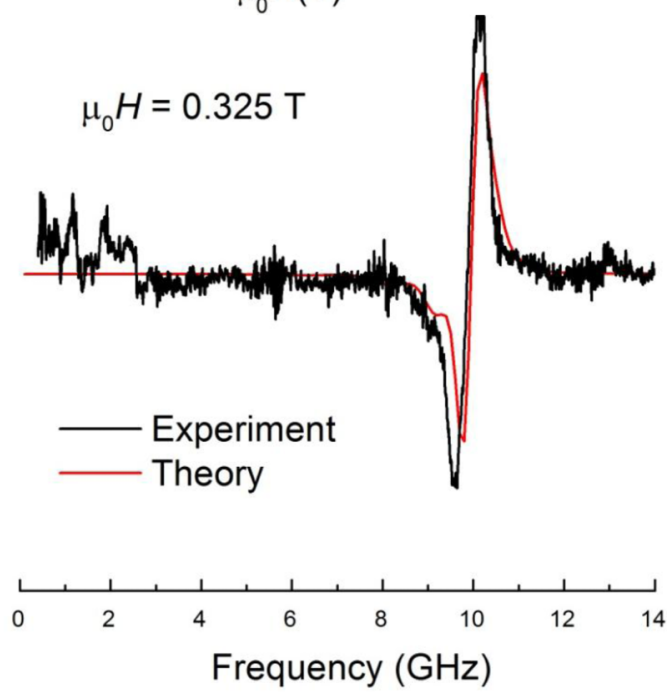

FIG. 2. Broadband magnetic spectroscopy with microwave transmission guides. Panels (a) and (b) are, respectively, experimental and calculated two-dimensional plots of the propagation of microwaves trough a coplanar superconducting transmission line that is coupled to a single crystal of $\mathrm{GdW}_{30}$ oriented as shown in the inset. The experiments were performed at $T=4.2 \mathrm{~K}$ and the magnetic field was applied along the $a$ crystallographic axis, close to the medium magnetic axis $x$. The calculations have been made using the spin Hamiltonian (1) and the magnetic anisotropy parameters given in the text. Panels (c) and (d) show traces obtained from these plots at, respectively, a fixed 10-GHz frequency as a function of magnetic field and at a fixed 0.325-T magnetic field as a function of frequency. These traces are indicated as arrows in (b). The resonant magnetic fields in (c) are $\mu_{0} H_{x}=0.340 \mathrm{~T}, 0.324 \mathrm{~T}, 0.319 \mathrm{~T}, 0.323 \mathrm{~T}, 0.325 \mathrm{~T}, 0.343 \mathrm{~T}$, and $0.423 \mathrm{~T}$ for transitions 1 to 7, respectively. The resonant frequencies in (d) are $\omega / 2 \pi=9.545 \mathrm{GHz}, 10.045 \mathrm{GHz}, 10.177 \mathrm{GHz}, 10.011 \mathrm{GHz}, 9.547 \mathrm{GHz}, 8.717 \mathrm{GHz}$, and $7.134 \mathrm{GHz}$ for transitions 1 to 7 , respectively.

however, that the decoherence induced by their couplings to the electronic spin is very weak [34] with $\mathrm{T}_{2, n}^{-1}<1 \mathrm{kHz}$. Therefore, the nuclear spin bath would limit the spin coherence within time scales of order of ms, which is much longer than the values of $\mathrm{T}_{2}$ measured experimentally. Spin coherence must, therefore, be limited by pair-flip processes [24] induced by residual dipolar interactions with other $\mathrm{GdW}_{30}$ molecules. The typical dipolar energy of each $\mathrm{GdW}_{30}$ molecule diluted in a $\mathrm{Y}_{0.99} \mathrm{Gd}_{0.01} \mathrm{~W}_{30}$ crystal is close to $4.5 \times 10^{-5} \mathrm{~K}=0.94 \mathrm{MHz}$ [18] and thus of the same order of magnitude as the experimental decoherence rates $\mathrm{T}_{2}^{-1}$. Coherence times of sufficiently well-isolated $\mathrm{GdW}_{30}$ molecules will therefore be limited only by the coupling of their spins to the lattice.
Spin-lattice relaxation times $\mathrm{T}_{1}$ have been determined by measuring the decay of the echo amplitude after a three-pulse sequence [31]. Values of $\mathrm{T}_{1}$ obtained for the different transitions, shown in Fig. 4(b), range from 2.3 to $2.8 \mu \mathrm{s}$ at $T=6 \mathrm{~K}$. While not extraordinarily long, the values of $\mathrm{T}_{2}$ and $\mathrm{T}_{1}$ nevertheless enable the coherent manipulation of the spin states.

\section{Rabi oscillations}

Spin nutation experiments were performed by measuring the spin echo amplitude generated by a variable duration excitation pulse (100 ns $\left.\leqslant t_{\mathrm{p}} \leqslant 1 \mu \mathrm{s}\right)$ followed, after a fixed time interval (typically between 120 and 200 ns), by 


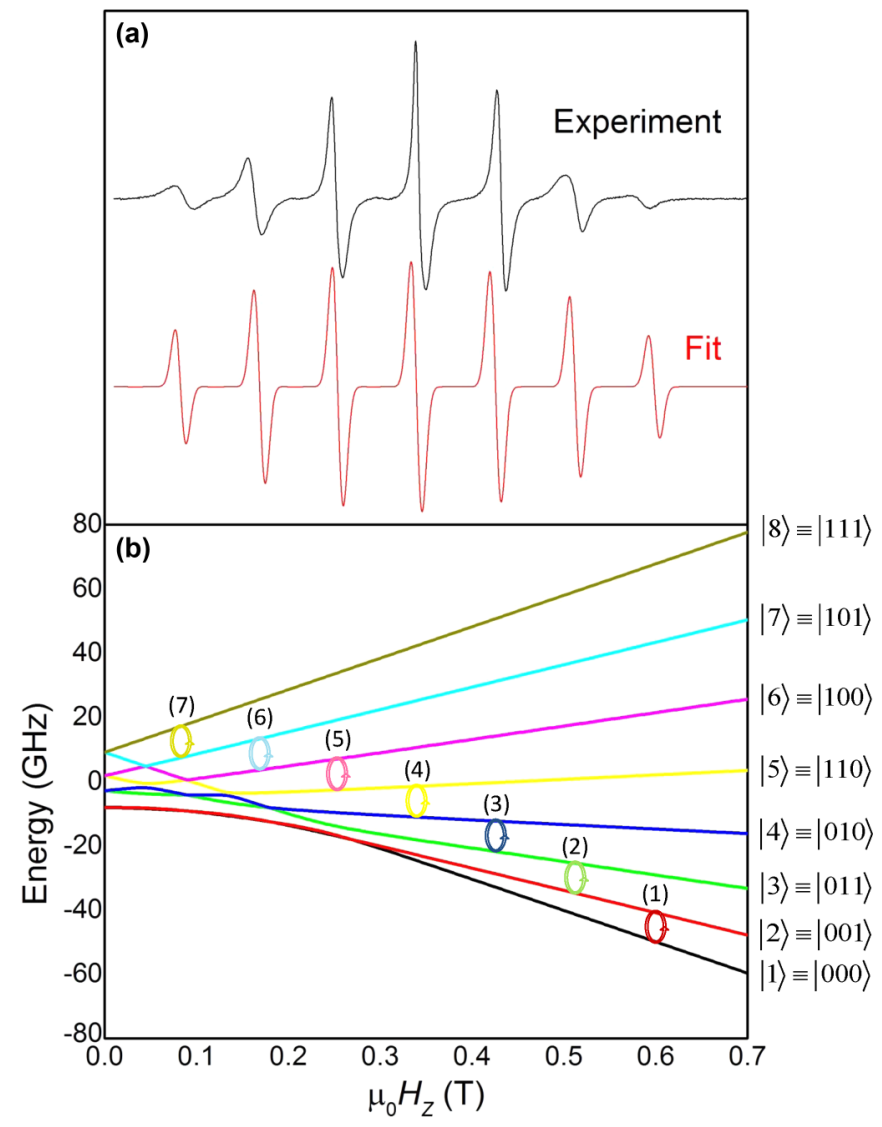

FIG. 3. (a) X-band cw-EPR spectrum measured at room temperature on a single crystal of $\mathrm{Y}_{0.95} \mathrm{Gd}_{0.05} \mathrm{~W}_{30}$. The crystal was rotated around its $a$ axis to maximize the field splitting of the seven transitions. (b) Zeeman diagram of the $\mathrm{GdW}_{30}$ spin energy levels vs. $H_{z}$. A possible assignment for the basis states of three qubits is shown. Arrows mark the seven addressable transitions between these states.

a refocusing $\pi$ pulse. Representative results obtained at the different resonant fields are shown in Fig. 5 and in Ref. [31]. For all transitions, $S_{y}$ shows damped coherent oscillations that are well described by the following expression (see Refs. [31] and [35] for a explicit derivation):

$$
S_{y, n}\left(t_{\mathrm{p}}\right)=K_{n} \exp \left(-t_{\mathrm{p}} / \tau_{\mathrm{R}, n}\right) J_{0}\left[\Omega_{\mathrm{R}, n}\left(t_{\mathrm{p}}-t_{\mathrm{p}, 0}\right)\right]+S_{y, 0}^{\mathrm{no}},
$$

where $K_{n}$ is a constant, $J_{0}$ is the Bessel function of first kind, $\Omega_{\mathrm{R}, n}$ and $1 / \tau_{\mathrm{R}, n}$ are, respectively, the frequency and the damping rate of each Rabi oscillation, $t_{\mathrm{p}, 0}$ accounts for the evolution at short times $t_{\mathrm{p}} \lesssim t_{m}$, and the last term is a nonoscillatory component. Values of $\Omega_{\mathrm{R}, n}$ and $1 / \tau_{\mathrm{R}, n}$ are shown in Fig. 5(b).

The frequencies of the coherent spin oscillations have been tuned by varying the microwave radiation power. The Rabi frequency increases linearly with $h_{1}$ (Fig. 6), in agreement with the theoretical expression $\Omega_{\mathrm{R}, n}=g \mu_{\mathrm{B}} a_{n} h_{1}$, where $a_{n}=$ $\left\langle n\left|2 S_{x}\right| n+1\right\rangle$ is the transition matrix element for a microwave magnetic field polarized along $x$. Figures 5(b) and 6 show that the experimental Rabi frequencies are, for any $h_{1}$, in very good agreement with those derived from Eq. (1). This

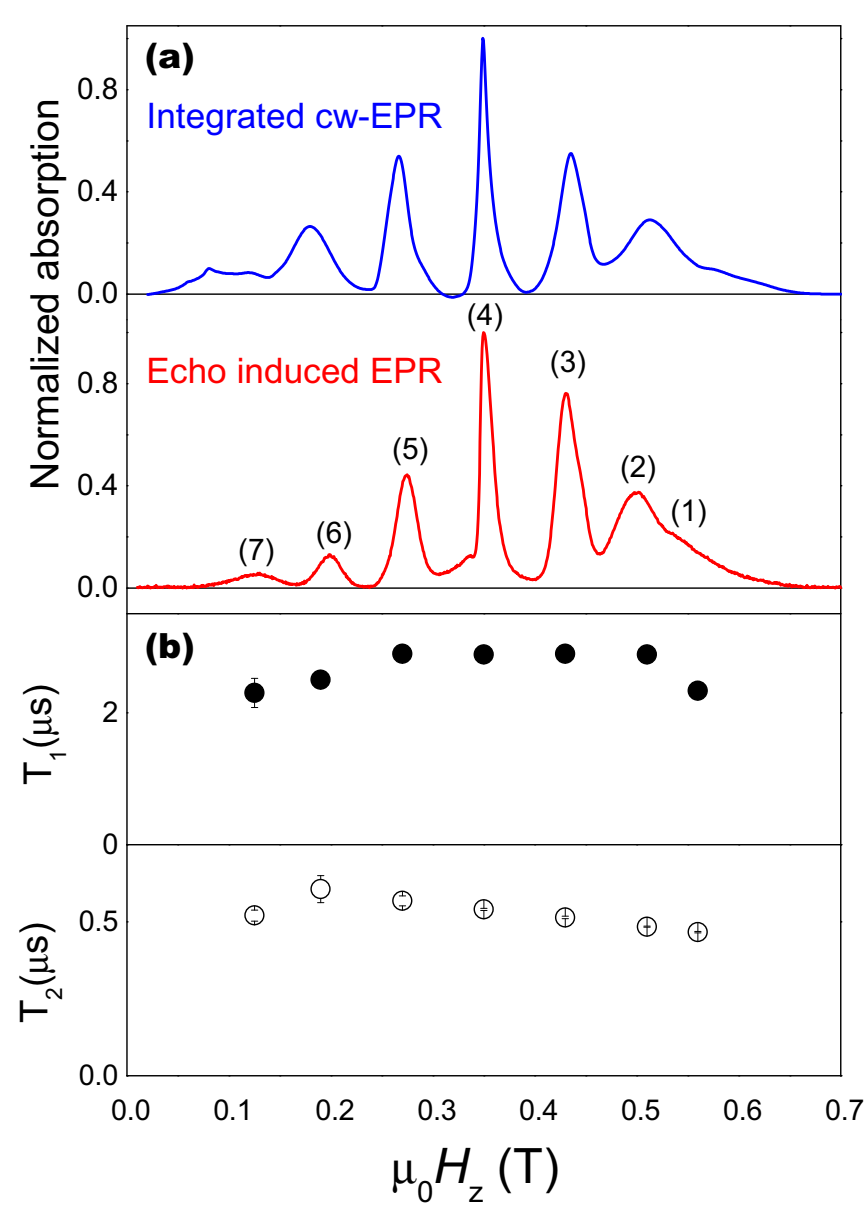

FIG. 4. (a) Comparison between the integrated cw-EPR spectrum and the two-pulse echo-induced EPR spectrum measured at $T=6 \mathrm{~K}$ on a $\mathrm{Y}_{0.99} \mathrm{Gd}_{0.01} \mathrm{~W}_{30}$ single crystal oriented as shown in Fig. 3(a). The interval between $\pi / 2$ and $\pi$ pulses was $\tau=160$ ns. (b) Spin coherence times $T_{2}$ and spin lattice relaxation $T_{1}$ times of transitions $n=7$ to 1 .

also shows that $\Omega_{\mathrm{R}, n}$ depends on $n$ but is independent of $H$, as far as the states involved can be approximated by pure spin projections. The number of observable oscillations does not increase with microwave power in the same manner as $\Omega_{\mathrm{R}, n}$ does, because the oscillation damping rate $1 / \tau_{\mathrm{R}, n}$ also increases with $h_{1}$ (Fig. 6). This effect limits the quantum quality factors to $\Omega_{\mathrm{R}, n} \tau_{\mathrm{R}, n} \simeq 5$, lower than the attainable limit $\Omega_{\mathrm{R}, n}\left(\mathrm{~T}_{2}^{-1}+\mathrm{T}_{1}^{-1}\right) / 2>30$. Such behavior is common with lanthanide spin qubits [27,28] and has been ascribed to inhomogeneities and fluctuations of the driving microwave field [36,37]. In the present case, fluctuations are likely associated with residual dipolar interactions between different molecular spins which limit the spin coherence times but can be minimized by dilution [18].

\section{Mapping to a three-qubit system}

The previous results show that each $\mathrm{GdW}_{30}$ molecule possesses eight well-defined spin states that can be coherently connected via seven separately addressable transitions. As Fig. 3(b) shows, the magnetic states can relabeled to map the basis states of three qubits. Within this scheme, the Rabi oscillations observed experimentally correspond to quantum 
(a)

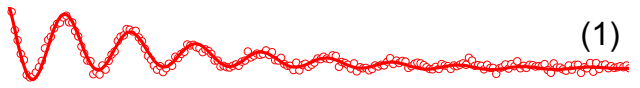

H. (2)

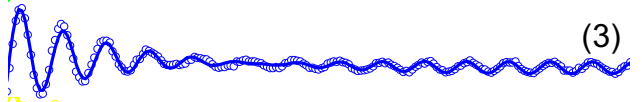

(4)

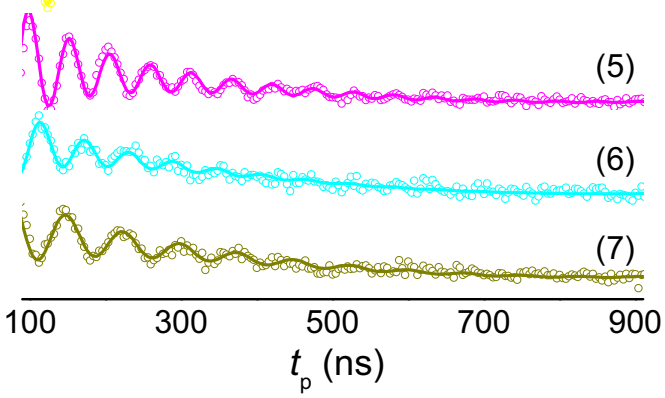

(b)

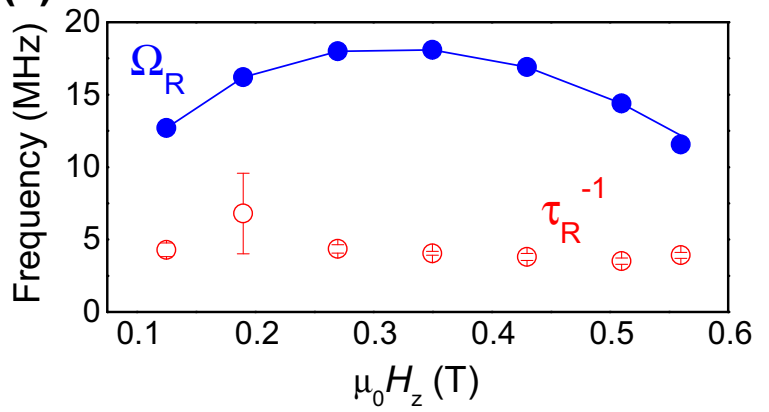

FIG. 5. (a) Rabi oscillations for transitions 1-7 measured on a $\mathrm{Y}_{0.99} \mathrm{Gd}_{0.01} \mathrm{~W}_{30}$ single crystal oriented as described in Fig. 3(a). The microwave magnetic field amplitude $\mu_{0} h_{1}=155(3) \mu \mathrm{T}$. Circles are experimental data and solid lines are least-squares fits based on Eq. (2). (b) Frequencies $\Omega_{\mathrm{R}, n}$ and damping rates $1 / \tau_{\mathrm{R}, n}$ of these oscillations. The solid blue line shows the theoretical $\Omega_{\mathrm{R}, n}$ derived from the spin Hamiltonian (1).

operations between two of these logic states. A relevant question to substantiate this mapping is whether these basic operations form a universal set, i.e., whether any quantum state can be generated by a combination of them. In the basis formed by the eigenstates $|n\rangle$ of Eq. (1) the coupling to an external radiation field can be described by the following effective Hamiltonian:

$$
\begin{aligned}
\mathcal{H}= & \sum_{n=1}^{8} \epsilon_{n}|n\rangle\langle n|-2 g \mu_{\mathrm{B}} h_{1} \cos (\omega t) \\
& \times \sum_{n=1}^{7}\left(a_{n}|n+1\rangle\left\langle n\left|+a_{n}^{*}\right| n\right\rangle\langle n+1|\right)
\end{aligned}
$$

that connects each level $n$ with its adjacent ones $n \pm 1$. Equation (3) contains the generators of a Lie algebra that includes all unitary operators in this Hilbert space of dimension $d=8$ and thus it is, by a suitable control of the $\mathrm{rf}$ fields $\vec{h}_{1}(t)$, capable of implementing any arbitrary quantum operations on the three qubits.

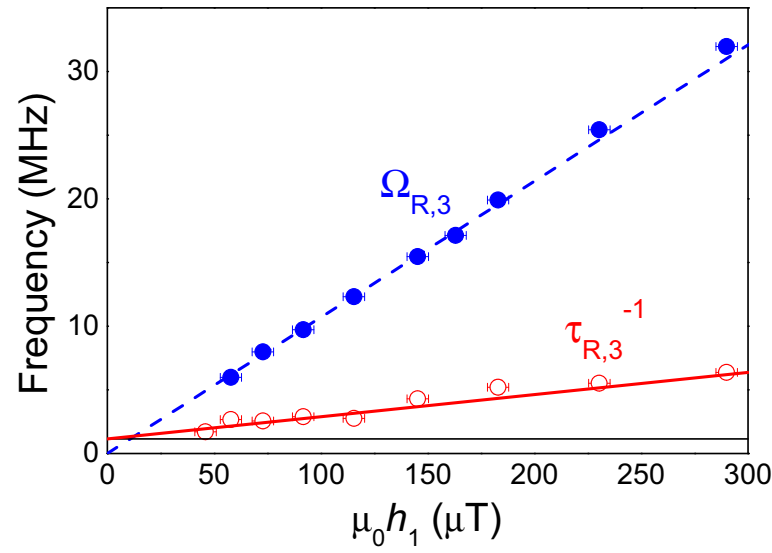

FIG. 6. Rabi frequency $\Omega_{R, 3}$ and damping rate $1 / \tau_{\mathrm{R}, 3}$ of transition 3 as a function of the microwave magnetic field amplitude $h_{1}$. The dashed blue line is the theoretical prediction derived from the spin Hamiltonian (1). The solid red line is a least-squares fit based on $1 / \tau_{\mathrm{R}}=\alpha+\beta \Omega_{\mathrm{R}}$ (see Ref. [31] for a discussion of this equation) while the solid horizontal line gives the low-power limit rate $\alpha=\left(1 / \mathrm{T}_{1}+1 / \mathrm{T}_{2}\right) / 2$ for this transition.

Universality must be complemented with specific protocols for designing actual quantum operations. Some can be realized by single pulses. An interesting example is a $\pi$ rotation $R_{1}(\pi)$ tuned at transition 1 . Because no other transition is resonant at the same frequency, this rotation realizes a controlled-controlled-NOT (CCNOT) or Toffoli gate [38] that flips the third "target" qubit if and only if the first two "control" qubits are both in state " 0 ." This idea is illustrated in Fig. 7, which shows the Rabi oscillation measured using the lowest microwave power, which corresponds to the lowest $\Omega_{\mathrm{R}, 1}$. In this plot, the implementation of a CCNOT gate corresponds to the coherent evolution generated by a $t_{\mathrm{p}} \simeq 84$-ns microwave pulse.

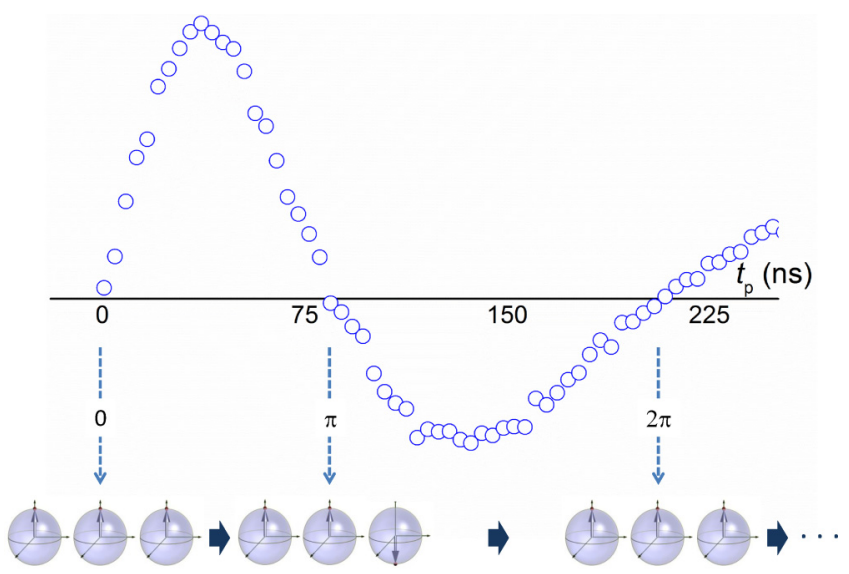

FIG. 7. Rabi oscillations for transition 1 measured on a $\mathrm{Y}_{0.99} \mathrm{Gd}_{0.01} \mathrm{~W}_{30}$ single crystal oriented as described in Fig. 3(a) for a microwave magnetic field amplitude $\mu_{0} h_{1}=52(3) \mu \mathrm{T}$. For clarity, the nonoscillatory part $\left[S_{y, 0}^{\text {no }}\right.$ in Eq. (2)] has been subtracted. The nonperfect periodicity is caused by the nonideal shape of short pulses. Using the labeling defined in Fig. 3(b), these oscillations correspond to a coherent evolution between $|000\rangle$ and $|001\rangle$ three-qubit states. The implementation of a CCNOT gate is then achieved by applying a resonant $\pi$ pulse of duration $t_{\mathrm{p}}=84 \mathrm{~ns}$, as illustrated in the figure. 
But, in general, complex operations involve also complex pulse sequences. For instance, starting from the ground state $|000\rangle$ the sequence $R_{1}(\pi / 2) R_{2}(\pi) R_{3}(\pi) R_{4}(\pi) R_{5}(\pi) R_{6}$ $(\pi) R_{7}(\pi)$, where $R_{n}(\theta)$ denotes a rotation by angle $\theta$ tuned at transition $n$, generates a maximally entangled state of the three qubits, namely the Greenberger-Horne-Zeilinger state $|\Psi\rangle=(1 / \sqrt{2})(|000\rangle+|111\rangle)$. Remarkably, this state is entangled in the logic basis but not in the spin basis, where it corresponds to a quantum superposition of the two states with maximum projections $\pm S$ along $\vec{H}$. This suggests that quantum correlations like those found in composite systems can also occur, and be experimentally tested, in a single magnetic ion. X-band cavities cannot apply pulses made of sufficiently different frequencies (at least $2 \mathrm{GHz}$ in the present case, see Fig. 2) [39]. Therefore, a different approach will be required to explore these phenomena. An option is to use the coupling to superconducting open wave guides, which, as shown in Fig. 2, give access to the relevant frequency range and which can be implemented in very low temperature environments. Information on the populations of different states can then be obtained via pump-probe experiments, measuring transmission near each resonant frequency $\omega_{n}$ and using the fact that only state $|n-1\rangle$ absorbs at this frequency.

\section{SUMMARY AND OUTLOOK}

Summarizing, the previous results show that single $\mathrm{Gd}^{3+}$ ions encapsulated in suitably chosen molecular environments provide model systems to investigate quantum dynamics of up to three qubits. Each $\mathrm{GdW}_{30}$ molecule provides a realization of a "qudit," that is, of a quantum system with $d=8$ fully controllable states. Although these properties might be achievable with ions in different coordinations [40], the molecular approach is particularly attractive for its versatility, since it can produce a vast choice of complexes encapsulating single magnetic ions with a spin $S>1 / 2$ and because it allows to fine-tune relevant parameters such as the magnetic anisotropy (that controls the span in resonance frequencies) [41]. It has been shown that nonclassical correlations [42,43], its relation to entropy [44], and even teleportation [45] can be explored in such quantum noncomposite systems.

Besides its fundamental interest, multilevel spin systems might potentially act as building blocks in scalable quantum computation schemes [46] because the molecules can be taken out of the crystal and placed individually on a surface or in a device [47]. The integration of multiple qubits in each molecule enhances the density of quantum information that could be handled with such schemes and reduces the number of nonlocal gates required to carry out any algorithm [14,48,49]. Another potential application concerns the implementation of quantum correction codes in each molecule [50-52]. Embedding qubits in higher-dimension qudits provides the possibility of restoring its quantum state from some specific errors [51]. A $\mathrm{GdW}_{30}$ molecular nanomagnet possesses the minimum dimension $d=8$ that is required to optimally correct a single amplitude or phase shift error. Besides, the fact that coherence times of all transitions are very close to each other, as shown by Figs. 4(b) and 5(b), ensures that errors occur with equal probability for all states. However, achieving a sufficiently strong coupling of single molecules to quantum superconducting circuits, which form the basis of some proposals [46], remains very challenging. Even for superconducting resonators with nanoscopic constrictions that locally enhance the microwave magnetic field [53], calculations suggest that single-photon coupling strengths $g_{n} \lesssim 0.05 \mathrm{MHz}[46,54]$ thus barely above the lowest decoherence rates reported for lanthanide qubits [22,27,28]. Enhancing the coupling of molecular nanomagnets to such circuits while, at the same time, minimizing decoherence is therefore one of the main challenges that need to be overcome in order to fully exploit the possibilities that these systems offer in terms of reproducibility, scalability, and design. Finally, we mention that the idea reported here can be extended to realize higher-dimension molecular qudits. The rational design of molecules containing two or three weakly coupled $\mathrm{Gd}^{3+}$ ions with distinct coordinations has been shown to be feasible [55]. Scaling up to six or nine addressable qubits within a molecule is therefore within reach.

\section{ACKNOWLEDGMENTS}

The authors acknowledge useful discussions with Seiji Miyashita, José Luis García-Palacios, and David Zueco. Funds were provided by the Spanish MINECO (Grants No. MAT2015-68204-R, No. CTQ2015-64486-R, No. MAT2014-56143-R, No. CTQ2014-52758-P, and No. FIS201570856-P and Excellence Unit María de Maeztu MDM-20150538), the European Union (ERC Grants No. SPINMOL and DECRESIM and COST 15128 Molecular Spintronics project), the Gobierno de Aragón (Grants No. E98-MOLCHIP and No. E33), Comunidad de Madrid (Research Network QUITEMAD+), and the Generalidad Valenciana (Prometeo and ISIC-Nano Programs of Excellence). A.G.-A. thanks MINECO for a Ramón y Cajal Fellowship.
[1] D. Gatteschi, R. Sessoli, and J. Villain, Molecular Nanomagnets (Oxford University Press, Oxford, 2006).

[2] Molecular Magnets, Physics and Applications, edited by J. Bartolomé, F. Luis, and J. F. Fernández (Springer - Verlag, Berlin, 2014).

[3] M. Mannini, F. Pineider, C. Danieli, F. Totti, L. Sorace, Ph. Sainctavit, M. A. Arrio, E. Otero, L. Joly, J. C. Cezar, A. Cornia, and R. Sessoli, Nature 468, 417 (2010).

[4] J. R. Friedman, M. P. Sarachik, J. Tejada, and R. Ziolo, Phys. Rev. Lett. 76, 3830 (1996)
[5] J. M. Hernández X. X. Zhang, F. Luis, J. Bartolomé, J. Tejada, and R. Ziolo, Europhys. Lett. 35, 301 (1996)

[6] L. Thomas, F. Lionti, R. Ballou, D. Gatteschi, R. Sessoli, and B. Barbara, Nature 383, 145 (1996).

[7] C. Sangregorio, T. Ohm, C. Paulsen, R. Sessoli, and D. Gatteschi, Phys. Rev. Lett. 78, 4645 (1997).

[8] S. Bertaina, S. Gambarelli, T. Mitra, B. Tsukerblat, A. Müller, and B. Barbara, Nature 453, 203 (2008).

[9] C. Schlegel, J. van Slageren, M. Manoli, E. K. Brechin, and M. Dressel, Phys. Rev. Lett. 101, 147203 (2008). 
[10] W. Wernsdorfer and R. Sessoli, Science 284, 133 (1999).

[11] E. Burzurí, F. Luis, O. Montero, B. Barbara, R. Ballou, and S. Maegawa, Phys. Rev. Lett. 111, 057201 (2013).

[12] A. Candini, G. Lorusso, F. Troiani, A. Ghirri, S. Carretta, P. Santini, G. Amoretti, C. Muryn, F. Tuna, G. Timco, E. J. L. McInnes, R. E. P. Winpenny, W. Wernsdorfer, and M. Affronte, Phys. Rev. Lett. 104, 037203 (2010).

[13] E. Burzurí, F. Luis, B. Barbara, R. Ballou, E. Ressouche, O. Montero, J. Campo, and S. Maegawa, Phys. Rev. Lett. 107, 097203 (2011).

[14] M. N. Leuenberger and D. Loss, Nature 410, 789 (2001).

[15] F. Troiani, A. Ghirri, M. Affronte, S. Carretta, P. Santini, G. Amoretti, S. Piligkos, G. Timco, and R. E. P. Winpenny, Phys. Rev. Lett. 94, 207208 (2005).

[16] A. Ardavan, O. Rival, J. J. L. Morton, S. J. Blundell, A. M. Tyryshkin, G. A. Timco, and R. E. P. Winpenny, Phys. Rev. Lett. 98, 057201 (2007).

[17] F. Luis, A. Repollés, M. J. Martínez-Pérez, D. Aguilà, O. Roubeau, D. Zueco, P. J. Alonso, M. Evangelisti, A. Camón, J. Sesé, L. A. Barrios, and G. Aromí, Phys. Rev. Lett. 107, 117203 (2011).

[18] M. J. Martínez-Pérez, S. Cardona-Serra, C. Schlegel, F. Moro, P. J. Alonso, H. Prima-García, J. M. Clemente-Juan, M. Evangelisti, A. Gaita-Ariño, J. Sesé, J. van Slageren, E. Coronado, and F. Luis, Phys Rev. Lett. 108, 247213 (2012).

[19] R. Vincent, S. Klyatskaya, M. Ruben, W. Wernsdorfer, and F. Balestro, Nature 488, 357 (2012).

[20] K. Bader, D. Dengler, S. Lenz, B. Endeward, S.-D. Jiang, P. Neugebauer, and J. van Slageren, Nat. Commun. 5, 5304 (2014).

[21] J. M. Zadrozny, J. Niklas, O. G. Poluektov, and D. E. Freedman, ACS Cent. Sci. 1, 488 (2015).

[22] M. Shiddiq, D. Komijani, Y. Duan, A. Gaita-Ariño, E. Coronado, and S. Hill, Nature 531, 348 (2016).

[23] N. V. Prokof'ev and P. C. E. Stamp, Rep. Prog. Phys. 63, 669 (2000).

[24] A. Morello, P. C. E. Stamp, and I. S. Tupitsyn, Phys. Rev. Lett. 97, 207206 (2006).

[25] F. Jelezko, T. Gaebel, I. Popa, A. Gruber, and J. Wrachtrup, Phys. Rev. Lett. 92, 076401 (2004).

[26] J. J. Pla, K. Y. Tan, J. P. Dehollain, W. H. Lim, J. J. L. Morton, D. N. Jamieson, A. S. Dzurak, and A. Morello, Nature 489, 541 (2012).

[27] S. Bertaina, S. Gambarelli, A. Tkachuk, I. N. Kurkin, B. Malkin, A. Stepanov, and B. Barbara, Nat. Nanotechnol. 2, 39 (2007).

[28] S. Bertaina, J. H. Shim, S. Gambarelli, B. Z. Malkin, and B. Barbara, Phys. Rev. Lett. 103, 226402 (2009).

[29] I. Creaser, M. Heckel, R. Neitz, and M. Pope, Inorg. Chem. 32, 1573 (1993).

[30] G. M. Sheldrick, SHELXTL Version 2014/7, http://shelx.uni-ac. gwdg.de/SHELX/index.php

[31] See Supplemental Material at http://link.aps.org/supplemental/ 10.1103/PhysRevB.95.064423 for further details on the sample structural and physical characterization and on the analysis of Rabi oscillations.
[32] A. Schweiger and G. Jeschke, Principles of Pulse Electron Paramagnetic Resonance (Oxford University Press, New York, 2001), Chap. 14.

[33] J. J. Baldoví, S. Cardona-Serra, J. M. Clemente-Juan, E. Coronado, A. Gaita-Ariño, and H. Prima-García, Chem. Commun. 49, 8922 (2013).

[34] S. Cardona-Serra, L. Escalera-Moreno, J. J. Baldoví, A. GaitaAriño, J. M. Clemente-Juan, and E. Coronado, J. Comp. Chem. 37, 1238 (2016).

[35] E. I. Baibekov, Appl. Magn. Reson. 45, 1289 (2014).

[36] H. De Raedt, B. Barbara, S. Miyashita, K. Michielsen, S. Bertaina, and S. Gambarelli, Phys. Rev. B 85, 014408 (2012).

[37] E. I. Baibekov, JETP Lett. 93, 292 (2011).

[38] A. Barenco, C. H. Bennett, R. Cleve, D. P. DiVincenzo, N. Margolus, P. Shor, T. Sleator, J. A. Smolin, and H. Weinfurter, Phys. Rev. A 52, 3457 (1995).

[39] Electron Spin Resonance (ESR) Based Quantum Computing, edited by T. Takui, L. Berliner, and G. Hanson (Springer, New York, 2016).

[40] E. I. Baibekov, M. R. Gafurov, D. G. Zverev, I. N. Kurkin, A. A Rodionov, B. Z. Malkin, and B. Barbara, Phys. Rev. B (to be published), arXiv:1610.04737 [cond-mat.mes-hall].

[41] Lanthanides and Actinides in Molecular Magnetism, edited by R. Layfield and M. Murugesu (Wiley-VCH, New York, 2015).

[42] A. A. Klyachko, M. A. Can, S. Binicioğlu, and A. S. Shumovsky, Phys. Rev. Lett. 101, 020403 (2008).

[43] R. Lapkiewicz, P. Li, C. Schaeff, N. K. Langford, S. Ramelow, M. Wieśniak, and A. Zeilinger, Nature 474, 490 (2011).

[44] E. O. Kiktenko, A. K. Fedorov, O. V. Man'ko, and V. I. Man'ko, Phys. Rev. A 91, 042312 (2015).

[45] E. O. Kiktenko, A. K. Fedorov, and V. I. Man'ko, Quantum Meas. Quantum Metrol. 3, 15 (2016).

[46] M. D. Jenkins, D. Zueco, O. Roubeau, G. Aromí, J. Majer, and F. Luis, Dalton Trans. 45, 16682 (2016).

[47] S. Sherif, G. Rubio-Bollinger, E. Pinilla-Cienfuegos, E. Coronado, J. C. Cuevas, and N. Agraï, Nanotechnology 26, 291001 (2015).

[48] B. P. Lanyon, M. Barbieri, M. P. Alemida, Th. Jenewein, T. C. Ralph, K. J. Resch, G. J. Pryde, J. L. O’Brien, A. Gilchrist, and A. G. White, Nat. Phys. 5, 134 (2009).

[49] E. O. Kiktenko, A. K. Fedorov, A. A. Strakhov, and V. I. Man'ko, Phys. Lett. A 379, 1409 (2015).

[50] P. Shor, Phys. Rev. A 52, R2493 (1995).

[51] S. Pirandola, S. Mancini, S. L. Braunstein, and D. Vitali, Phys. Rev. A 77, 032309 (2008).

[52] J. J. Baldoví, S. Cardona-Serra, J. M. Clemente-Juan, L. Escalera-Moreno, A. Gaita-Ariño, and G. Mínguez-Espallargas, Europhys. Lett. 110, 33001 (2015).

[53] M. Jenkins, U. Naether, M. Ciria, J. Sesé, J. Atkinson, C. Sánchez-Azqueta, E. del Barco, J. Majer, D. Zueco, and F. Luis, Appl. Phys. Lett. 105, 162601 (2014).

[54] M. Jenkins, T. Hümmer, M. J. Martínez-Pérez, J. J. GarcíaRipoll, D. Zueco, and F. Luis, New J. Phys. 15, 095007 (2013).

[55] D. Aguilà, L. A. Barrios, V. Velasco, O. Roubeau, A. Repollés, P. J. Alonso, J. Sesé, S. J. Teat, F. Luis, and G. Aromí, J. Am. Chem. Soc. 136, 14215 (2014). 